\section{Response and resistance to CDK12 inhibition in aggressive B-cell lymphomas}

\author{
Jing Gao, ${ }^{1, *}$ Michelle Y. Wang, ${ }^{1, *}$ Yuan Ren, ${ }^{1}$ Tint Lwin,,${ }^{1}$ Tao Li, ${ }^{1}$ Joy C. Yan, ${ }^{1}$ \\ Eduardo M. Sotomayor, ${ }^{2}$ Derek R. Duckett, ${ }^{1}$ Bijal D. Shah, ${ }^{3}$ Kenneth H. Shain, ${ }^{3}$ \\ Xiaohong $\mathrm{Zhao}^{1}$ and Jianguo $\mathrm{Tao}^{4}$
}

${ }^{1}$ Chemical Biology and Molecular Medicine Program, H. Lee Moffitt Cancer Center \& Research Institute, Tampa, FL; ${ }^{2}$ Cancer Institute, Tampa General Hospital,Tampa, FL; ${ }^{3}$ Departments of Malignant Hematology, H. Lee Moffitt Cancer Center \& Research Institute, Tampa, FL and ${ }^{4}$ (Previous affiliation) Department of Hematopathology and Laboratory Medicine, H. Lee Moffitt Cancer Center \& Research Institute, Tampa, FL, USA

*JG and MYW contributed equally as co-first authors.

\section{ABSTRACT}

$\mathrm{D}$ espite significant progress in the treatment of patients with diffuse large B-cell lymphoma (DLBCL) and mantle cell lymphoma $(\mathrm{MCL})$, the prognosis of patients with relapsed disease remains poor due to the emergence of drug resistance and subsequent disease progression. Identification of novel targets and therapeutic strategies for these diseases represents an urgent need. Here, we report that both MCL and DLBCL are exquisitely sensitive to transcription-targeting drugs, in particular THZ531, a covalent inhibitor of cyclin-dependent kinase 12 (CDK12). By implementing pharmacogenomics and a cell-based drug screen, we found that THZ531 leads to inhibition of oncogenic transcriptional programs, especially the DNA damage response pathway, MYC target genes and the mTOR-4EBP1-MCL-1 axis, contributing to dramatic lymphoma suppression in vitro. We also identified de novo and established acquired THZ531-resistant lymphoma cells conferred by over-activation of the MEK-ERK and PI3K-AKT-mTOR pathways and upregulation of multidrug resistance-1 (MDR1) protein. Of note, EZH2 inhibitors reversed resistance to THZ531 by competitive inhibition of MDR1 and, in combination with THZ531, synergistically inhibited MCL and DLBCL growth in vitro. Our study indicates that CDK12 inhibitors, alone or together with EZH2 inhibitors, offer promise as novel effective approaches for difficult-to-treat DLBCL and MCL.

\section{Introduction}

MYC is a transcription factor that promotes oncogenesis by activating and repressing its target genes that control cell growth and proliferation. ${ }^{1}$ While MYC has been described as a defining feature and the driving oncogene for Burkitt lymphoma, the significance of MYC has also been recognized in other non-Hodgkin lymphomas. ${ }^{2}$ MYC, which has been detected in 15-20\% of diffuse large B-cell lymphomas (DLBCL), is associated with an adverse prognosis as a result of chemoresistance and, shortened survival. In mantle cell lymphoma (MCL), increased expression of MYC has been found to be associated with poor prognosis and more aggressive disease. ${ }^{3}$ MYC overexpression has also been implicated in high-grade large cell transformation. ${ }^{4}$ Despite current modes of intensive chemotherapy and immunotherapy, MCL and other MYC-associated lymphomas are aggressive diseases that respond poorly to chemoimmunotherapy and affected patients have a dismal survival. Identification of effective strategies to target these aggressive lymphomas represents an urgent need.

Recently, we integrated data from our unbiased activity-based proteomic profiling, RNA-sequencing, and chromatin immunoprecipitation (ChIP) assays with sequencing studies. We found that the aggressive progression and development of drug resistance in MCL and MYC-associated lymphomas require complex transcriptome and kinome remodeling of cellular signaling networks, positive feedback loops that amplify pro-survival and growth signals. ${ }^{5}$ Rather than there being a single mechanism
Ferrata Storti Foundation

Haematologica 2022

Volume 107(5):1119-1130

\section{Correspondence:}

JIANGUO TAO

dtep642021@gmail.com

Received: March 10, 2021.

Accepted: June 10, 2021.

Pre-published: June 24, 2021.

https://doi.org/10.3324/haematol.2021.278743

(c)2022 Ferrata Storti Foundation

Material published in Haematologica is covered by copyright. All rights are reserved to the Ferrata Storti Foundation. Use of published material is allowed under the following terms and conditions:

https://creativecommons.org/licenses/by-nc/4.0/legalcode. Copies of published material are allowed for personal or internal use. Sharing published material for non-commercial purposes is subject to the following conditions:

https://creativecommons.org/licenses/by-nc/4.0/legalcode, sect. 3. Reproducing and sharing published material for commercial purposes is not allowed without permission in writing from the publisher. 
of driving lymphoma progression, networks are rewired and, consequently, the signal rewiring must be targeted as a whole to obtain durable and improved clinical responses. In support of this concept, increasing evidence suggests that super-enhancers are required to maintain the expression of genes critical for cancer cell survival and proliferation. Given the biological importance of super-enhancers in the regulation of global transcriptome landscaping, superenhancers have emerged as enticing therapeutic targets. Indeed, blocking super-enhancer-driven transcription resulted in the concurrent disruption of multiple oncogenic machineries to which aggressive lymphoma cells are addicted, regardless of their mutational profiles. ${ }^{6,7}$ Thus, small-molecule inhibitors targeting transcriptional regulators and downstream global transcriptome and kinome reprogramming represent a promising approach to the treatment of aggressive lymphomas.

Transcriptional cyclin-dependent kinase 7 (CDK7) and 9 (CDK9), catalytic subunits of the transcription elongation factor P-TEFb, are considered to be gatekeepers of the transcriptional machinery, ${ }^{8}$ and clinical trials with small-molecule inhibitors of these kinases are underway. ${ }^{9} \mathrm{CDK} 7-$ and CDK9-mediated phosphorylation of serine on the C-terminal domain of RNA polymerase II (RNAPII) is known to be linked to transcriptional initiation and elongation. Phosphorylation of Ser2 on the C-terminal domain is catalyzed by P-TEFb and is associated with a large complex of proteins coined the super elongation complex.10,11 Moreover, CDK7 directly phosphorylates Ser5 and Ser7 of the C-terminal domain of RNAPII for RNAPII activity. Interestingly, ChIP-sequencing data demonstrated that CDK7 densely occupies super-enhancers that drive high levels of transcription of oncogenes, such as MYC, in a wide variety of cancers, including T-cell acute lymphoblastic leukemia, nonsmall cell lung cancer, neuroblastoma and triple-negative breast cancer. 12-14 Importantly, treatment of cancer with a selective, small-molecule CDK7 inhibitor, THZ1, leads to rapid loss of these super-enhancer-driven oncogenic transcripts ${ }^{15}$ suggesting that super-enhancer-driven genes are especially vulnerable to inhibition of transcriptional machinery such as through inhibition of CDK7. ${ }^{16}$

CDK12 belongs to the transcriptional CDK family of serine/threonine protein kinases that regulate transcriptional and post-transcriptional processes, thereby modulating multiple cellular functions. CDK12 modulates transcription elongation by phosphorylating the Ser2 on the C-terminal domain of RNAPII and was demonstrated to specifically upregulate the expression of genes involved in the DNA damage response (DDR), mRNA processing, stress and heat shock. ${ }^{17}$ Other studies indicated that CDK12 phosphorylates pre-mRNA processing factors directly, thereby inducing premature cleavage and polyadenylation and a loss of expression of long genes $(>45 \mathrm{~kb})$, a substantial proportion of which participate in the DDR. ${ }^{18,19}$ In addition, an increasing number of studies point to CDK12 inhibition as an effective strategy to inhibit tumor growth, and synthetic lethal interactions have been described with MYC, EWS/FLI and PARP/CHK1 inhibition. ${ }^{20,21}$ Therefore, CDK12 has emerged as an appealing therapeutic target.

MCL is a B-cell malignancy in which the disruption of the DDR pathway and activation of cell survival mechanisms contribute to oncogenesis. ${ }^{22}$ MYC activation engages the replication stress and DNA damage pathway to allow not only robust cellular proliferation, but also limited clonal expansion and avoidance of cytotoxic DNA damage accu- mulation in aggressive B-cell lymphomas. ${ }^{23,24}$ These data implicate CDK12 as a potential novel vulnerability for MCL and MYC-associated large B-cell lymphomas.

In this study, we determined the role of CDK12-mediated transcriptional activation and its associated pathway in cell survival and growth in MCL and MYC-associated large Bcell lymphomas. We defined the molecular mechanism for inhibiting CDK12 using THZ531 in these aggressive lymphomas. Importantly, we investigated the molecular mechanism conferring resistance to THZ531 and examined whether combined inhibitors of CDK12 and EZH2 cooperatively reprogram transcriptional repression to overcome THZ531 resistance, and, ultimately, inhibit lymphoma growth and survival in these difficult-to-treat, aggressive Bcell malignancies.

\section{Methods}

\section{Patients and tumor specimens}

The primary samples from MCL patients were obtained from fresh biopsy-derived lymphoma tissues (lymph nodes) and from peripheral blood following informed consent from patients and approval by the Moffitt Cancer Center/University of South Florida Institutional Review Board. For preparation of viable, sterile, single-cell suspensions, the lymph node tissue was diced and forced through a cell strainer into RPMI-1640 tissue culture medium. Cells, obtained after low-speed centrifugation were re-suspended in medium. Lymphoma cells from peripheral blood were isolated by Ficoll-Plaque purification, and only lymphoma samples that had more than $80 \%$ tumor cells were used for experiments.

\section{Image-based cell-viability assay}

Cells were seeded in a 384-well plates of a reconstructed lymphoma tumor microenvironemnt, including high physiological densities $\left(1-10 \times 10^{6}\right.$ cells $/ \mathrm{mL}$ ), extracellular matrix (collagen, Advanced BioMatrix, \#5005-B), and lymphoma stromal cells. A panel of drugs at five serial diluted concentrations was added to the medium, and plates were continuously imaged every $30 \mathrm{~min}$ for $96 \mathrm{~h}$ (cell lines) or $144 \mathrm{~h}$ (primary samples). All images were analyzed using a digital imaging analysis algorithm to detect cell viability based on membrane motion (pseudo-colored in green), and changes in viability were quantified by the area under the curve (AUC) as described elsewhere..$^{25-27}$

\section{RNA-sequencing}

All samples were prepared in biological triplicates. Cells $\left(10 \times 10^{6}\right)$ were treated with $100 \mathrm{nM}$ THZ531 or dimethylsulfoxide (vehicle control) for 6 and $24 \mathrm{~h}$. Total RNA was isolated using an RNA isolation kit, RNeasy Plus Mini (Qiagen Cat\# 74134). Libraries were prepared using a TruSeq Stranded mRNA Library Prep Kit (Illumina Cat \#RS-122-2101/2) according to the manufacturer's instructions. RNA sequencing was performed on a HiSeq $2500 \mathrm{v} 4$ high output (50 bp, single-end reads). Tophat2 was used to align the Fastq files. Transcripts per kilobase million (TPM) values were calculated and normalized using Cuffnorm. Genes that had a $P$ less than 0.05 and at least a 1.5 -fold change were considered to be significantly altered between sensitive and resistant phenotypes. The cutoff value for expressed genes was a TPM value greater than or equal to 1 .

\section{Statistics}

Unless otherwise stated, comparisons and statistical significance between two groups in this paper are based on a two-sided Student $t$-test. $P$ values of less than 0.05 were considered statisti- 
cally significant. Data are shown with the mean \pm standard deviation of at least three experiments. Analysis of variance (ANOVA) or the Kruskal-Wallis test was used for comparing data from multiple groups.

\section{Results}

\section{Mantle cell lymphoma and other B-cell lymphoma cell lines and primary samples are exquisitely sensitive to CDK12 inhibition regardless of genetic background and drug resistance status}

We performed cell viability assays on over $40 \mathrm{~B}$-cell lymphoma lines for their response to the CDK12 inhibitor THZ531. These cell lines included MCL, DLBCL, double hit lymphoma and Burkitt lymphoma lines with a variety of genetic backgrounds. Established ibrutinib-resistant lines were also included for their vulnerability to CDK12 inhibition. As shown in Figure 1A and Online Supplementary Figure $S 1 A$, most of these cell lines exhibited high sensitivity to THZ531 regardless of lymphoma type, genetic background (11q, p53 status) and drug resistance with all $\mathrm{IC}_{50}$ values in the nanomolar range except for REC-1. In parallel, we also performed western blotting on all tested lines to determine the relative protein abundances of MCL-1, 4EBP1 and its phosphorylation (Online Supplementary Figure S1B) and the correlation of protein abundances with THZ531 sensitivity. As shown in Figure 1B, THZ531 IC I0 $_{50}$ values were most correlated to the protein levels of MCL-1 and phosphorylated 4EBP1. Furthermore, primary MCL samples were exquisitely sensitive to THZ531 (Figure 1C), implying that CDK12 has a functional role in aggressive B-cell malignancies. We therefore queried CDK12 gene expression from RNAsequencing performed on 40 primary samples. Notably, the expression level of MCL-1 and DDR-related genes correlated positively with CDK12 mRNA expression in B-cell lymphomas (Figure 1D, Online Supplementary Figure S1C). Therefore, CDK12 expression is frequently elevated and is essential for cell growth and survival in MCL and aggressive B-cell lymphomas.

\section{CDK12 sustains cell growth and survival through transcriptional activation of MYC, the mTOR-4EBP1-MCL-1 axis and DNA damage response pathway in mantle cell lymphoma and MYC-associated B-cell lymphomas}

The strong correlations of sensitivity to THZ531 and MCL-1 protein level and 4EBP1 phosphorylation indicated the functional role of the mTOR-4EBP1-MCL-1 axis in the growth and survival of these aggressive lymphomas. We next examined the effect of CDK12 inhibition with THZ531 on cellular signaling molecules such as those involved in the PI3K-AKT-mTOR pathway in addition to MCL-1 in MCL (Z138, Jeko-1), double-hit lymphoma (DOHH-2), DLBCL (Val) cell lines and primary MCL samples. Given that CDK12 is one of the transcriptional CDK considered to be a gatekeeper of transcriptional elongation, the effects of THZ531 on phosphorylation of Ser2 on the C-terminal domain of RNAPII, MYC, the AKT-mTOR4EBP1 pathway, BCL-2 family proteins, and apoptotic PARP cleavage were assessed by western blot. As shown in Figure 2A and Online Supplementary Figure S2A, THZ531 induced a dose-dependent inhibition of RNAPII-Ser2 phosphorylation and variable degrees of inhibition of RNAPIISer5/7 phosphorylation at higher doses with associated dramatic reductions of MYC and MCL-1 protein levels and triggered significant PARP cleavage in all the tested lymphoma lines. In addition, phosphorylation of p70S6K and 4EBP1, the downstream targets of mTOR, were also markedly decreased after THZ531 treatment (Figure 2A, Online Supplementary Figure S2A). Together, these results, in conjunction with the correlative studies shown in Figure $1 \mathrm{~B}$, imply that the mTOR-4EBP1 pathway and subsequent cap-dependent translation targets, MCL-1 and MYC, mediate CDK12 function in these aggressive B-cell lymphomas. To confirm this notion, we employed the 4EBP1 mutant, 4EBP1, as a dominant cap-dependent translation initiation inhibitor. ${ }^{28}$ 4EBP1 carries alanine substitutions at four serine/threonine residues and thereby prevents dissociation of 4EBP1 from eIF4E induced by mTORC1. The abundance of MYC and MCL-1 proteins was decreased upon ectopic expression of doxycycline-induced 4EBP1 in HBL-2 cells (Figure 2B). Interestingly, when cells with ectopic expression of doxycycline-induced 4EBP1 were treated with THZ531, we observed more dramatic reductions in MCL1, MYC, and cell viability with associated increased PARP cleavage relative to non-induced cells (Figure 2C, Online Supplementary Figure S2B). This enhanced effect of THZ531 is likely attributable to endogenous translation activity. Moreover, in line with these results, increased ectopic expression of MCL-1 attenuated THZ531-induced MCL-1 downregulation, apoptosis and colony formation (Figure 2D, E, Online Supplementary Figure S2C). Together, these results indicate that 4EBP1 and MCL-1 mediate, at least partially, the biological function of CDK12.

To determine the molecular mechanism and cellular pathways responsible for the activity of THZ531, RNAsequencing was performed on THZ531-sensitive MCL lines (Z138, Jeko-1) that were treated or not with $100 \mathrm{nM}$ THZ531 for 6 and $24 \mathrm{~h}$. Transcriptomic analysis of these THZ531-treated cells revealed alteration of expression of a large set of genes, with a total of 2,405 genes downregulated in common in the two cell lines after $6 \mathrm{~h}$ and 2,914 genes downregulated in common after $24 \mathrm{~h}$, implying alteration of similar pathways (Figure 3A, Online Supplementary Figure S3A). Gene set enrichment analysis revealed that genes altered by THZ531 treatment for $24 \mathrm{~h}$ are associated with proliferation and survival pathways as well as both the MYC and mTOR-AKT pathways (Figure 3B, Online Supplementary Figure S3B). Further analysis using the Kyoto Encyclopedia of Genes and Genomes (KEGG) revealed several DDR pathways significantly negatively enriched by $24 \mathrm{~h}$ of treatment, including homologous recombination and the mismatch-repair pathways (Figure 3C, Online Supplementary Figure S3C). Overall, these data support THZ531-mediated transcriptional suppression.

In parallel, we performed drug screening in cells sensitive to THZ531 treatment and determined their drug response profiles. As shown in Figure 3D, the MCL and aggressive B-cell lymphoma lines were uniformly sensitive to inhibitors of the transcriptional machinery apparatus (CDK7, CDK9 and CDK12), AKT, mTOR, and PLK, as well as the chemotherapeutic drug doxorubicin. Intriguingly, each of these inhibitors and chemotherapeutic drugs is known to function by downregulating MCL-1, and ectopic overexpression of MCL-1 restored the potency of these agents (Figure 3E), thus supporting the role of MCL-1 in cell survival and drug response to CDK12 inhibition. In line with these cell line results, when we performed RNAsequencing on primary MCL samples after treatment with 
A

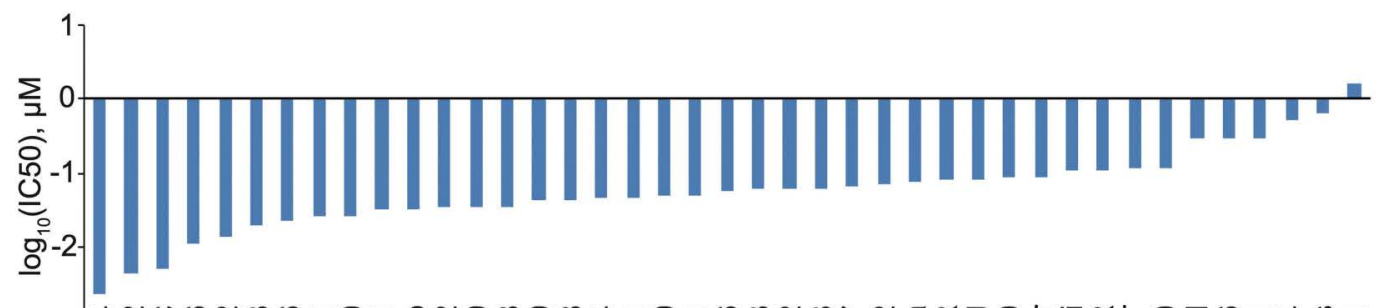

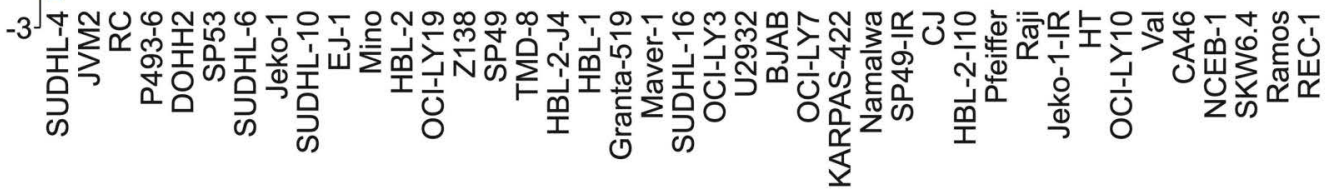

B
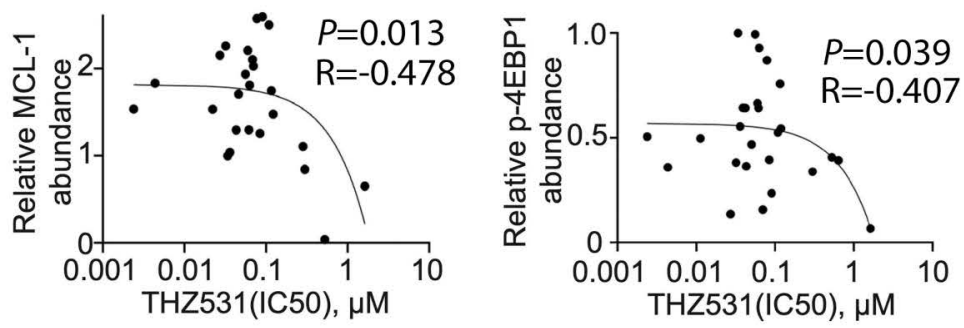

C
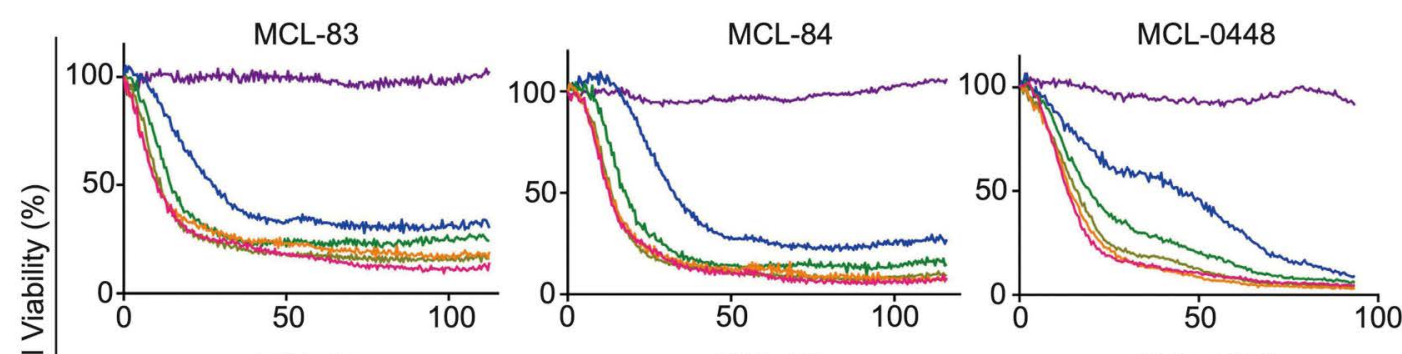

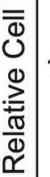
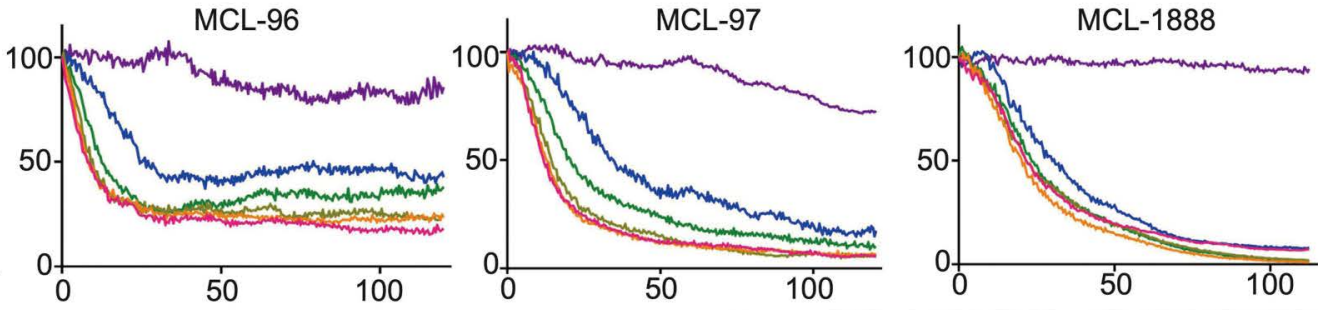

Time (h)

THZ531: $-3 \mu \mathrm{M}-1 \mu \mathrm{M}-0.33 \mu \mathrm{M}-0.11 \mu \mathrm{M}-0.04 \mu \mathrm{M}-\mathrm{DMSO}$

D
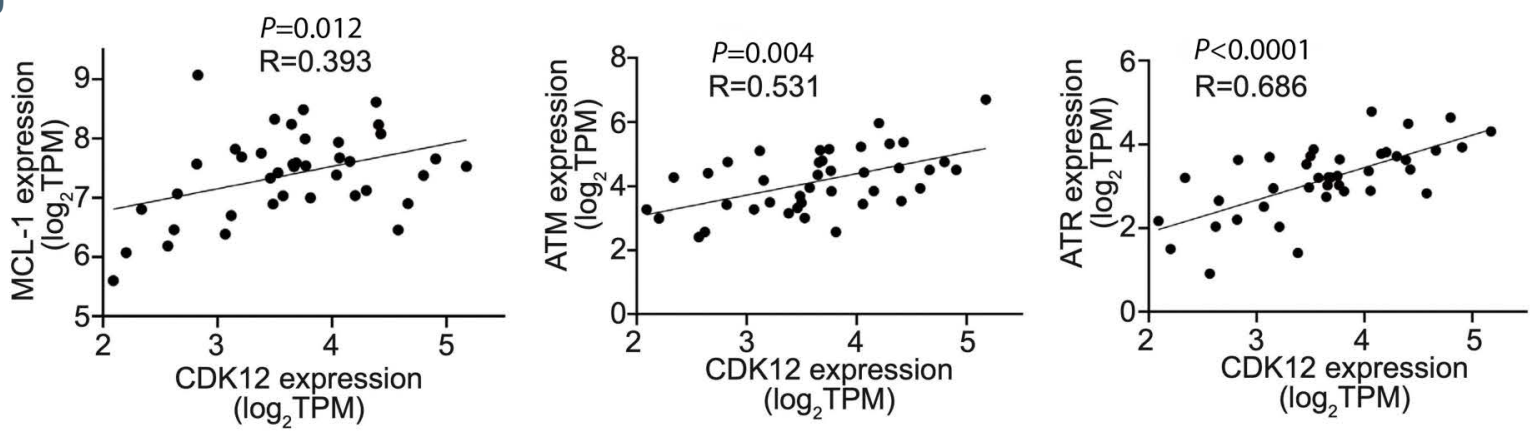

Figure 1. Mantle cell lymphoma and other B-cell lymphoma cell lines and primary samples are exquisitely sensitive to CDK12 inhibition with THZ531, regardless of genetic background and drug resistance status. (A) Bar plot of $\log _{10}$ of $50 \%$ inhibitory concentration (IC 50 ) of B-cell lymphoma cell lines treated with THZ531 for $72 \mathrm{~h}$. (B) Correlation of MCL-1 (left) or phosphor-4EBP1 protein level (right) with THZ531 IC $_{50}$ in B-cell lymphoma cell lines. (C) Image-based cell-viability assays of primary mantle cell lymphoma samples; $3 \times 10^{6}$ cells were seeded in a 384-well plate with extracellular matrix and lymphoma stromal cells. THZ531 at five serial diluted concentrations was added to the medium, and the plate was continuously imaged every 30 min for $144 \mathrm{~h}$. All images were analyzed using a digital imaging analysis algorithm to detect cell viability based on membrane motion, and changes in viability were quantified by area under the curve (AUC). (D) Correlation of MCL-1 (left), ATM (middle) and ATR (right) gene expression ( $\log _{2}$ TPM) with CDK12 gene expression $\left(\log _{2}\right.$ TPM) in primary patients' samples. Data shown in (A) are representative of at least three independent experiments. TPM: transcripts per kilobase million. 
100 or $500 \mathrm{nM}$ THZ531 for $12 \mathrm{~h}$, we observed that gene signatures related to the MYC, mTOR and DDR pathways were significantly downregulated (Figure 3F, Online Supplementary Figure S3D). These results support the functional role of CDK12 in MCL survival and proliferation.
MDR1 upregulation drives resistance to THZ531 in mantle cell lymphoma and other aggressive

B-cell lymphomas

Despite the potency shown by THZ531 and other transcriptional CDK inhibitors in preclinical studies, resistance
A

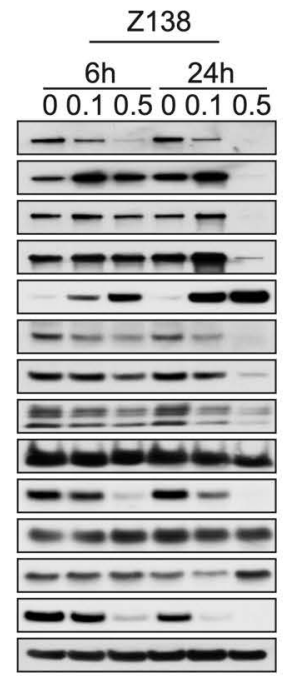

B

HBL-2

4EBP1

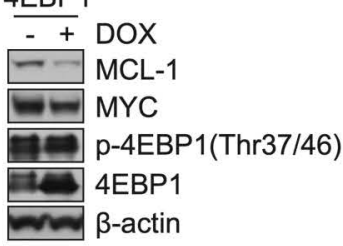

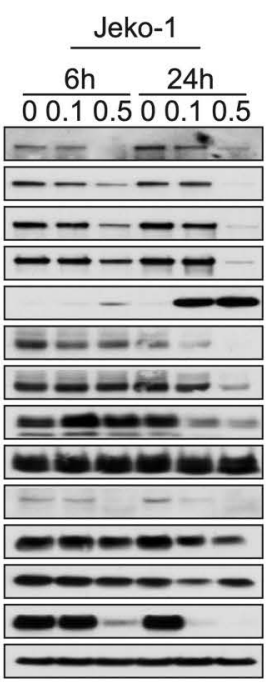

\begin{tabular}{|c|c|}
\hline MCL-0448 & \\
\hline $24 \mathrm{~h}$ & \\
\hline$\overline{00.10 .5} \overline{00.10 .5}$ & THZ531 $(\mu \mathrm{M})$ \\
\hline$--\cdots-\cdots$ & p-Ser2 RNAPII \\
\hline----- & p-Ser5 RNAPII \\
\hline$----\cdots$ & p-Ser7 RNAPII \\
\hline----- & Total RNAPII \\
\hline$-\cdots$ & cleaved PARP \\
\hline----1 & p-p70S6K(Ser371) \\
\hline----- & p70s6K \\
\hline$-0-0=$ & p-4EBP1(Thr37/46) \\
\hline ecteve & 4EBP1 \\
\hline$--\cdots$ & MCL-1 \\
\hline----- & BCL-XL \\
\hline - & BCL-2 \\
\hline--1 & MYC \\
\hline & $\beta$-actin \\
\hline
\end{tabular}

HBL-2

C

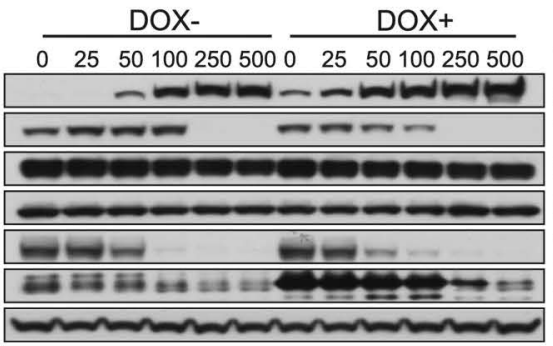

THZ531 (nM)

cleaved PARP

$M C L-1$

$B C L-X L$

$B C L-2$

MYC

4EBP1

$\beta$-actin

D

E

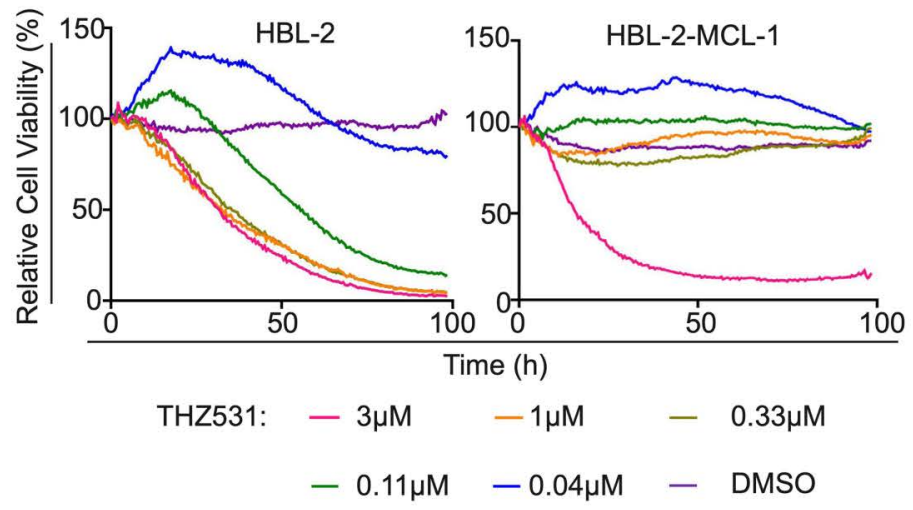

HBL-2 HBL-2-MCL-1 $0 \longdiv { 2 5 5 0 1 0 0 } 0 2 5 5 0 1 0 0$ THZ531 (nM)

cleaved PARP
QE-EDETMC

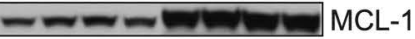
BCL-XL $\beta$-actin

Figure 2. CDK12 sustains cell growth and survival through transcriptional activation of MYC and the mTOR-4EBP1-MCL-1 axis in mantle cell lymphoma and MYCassociated B-cell lymphomas. (A) Western blot analysis of phosphorylation of RNAPII in Ser2, 5 and 7, RNAPII, cleaved PARP, phosphor-p70S6K, p70S6K, phosphor4EBP1, 4EBP1, MCL-1, BCL-XL, BCL-2 and MYC in THZ531 sensitive cell lines and mantle cell lymphoma primary samples treated with the indicated doses of THZ531 at different time points. (B) Western blot analysis of MCL-1, MYC, phosphor-4EBP1 and 4EBP1 protein levels in HBL-2 cells with and without doxycycline-induced overexpression of 4EBP1. (C) Western blot analysis of cleaved PARP, MCL-1, BCL-XL, BCL-2, MYC and 4EBP1 protein expression in HBL-2 cells with and without doxycycline-induced overexpression of 4EBP1 treated with the indicated doses of THZ531 for $24 \mathrm{~h}$. (D) Image-based cell-viability assays of HBL-2 cells with and without overexpression of MCL-1. Cells $\left(1 \times 10^{6}\right)$ were seeded in a 384-well plate with extracellular matrix. THZ531 was added at five serial diluted concentrations to the medium, and the plate was continuously imaged every $30 \mathrm{~min}$ for $96 \mathrm{~h}$. All images were analyzed using a digital imaging analysis algorithm to detect cell viability based on membrane motion, and changes in viability were quantified by the area under the curve. (E) Western blot analysis of cleaved PARP, MYC, MCL-1 and BCL-XL in HBL-2 cells with and without MCL-1 overexpression treated with the indicated doses of THZ531 for $24 \mathrm{~h}$. DOX: doxycycline; DMSO: dimethylsulfoxide. 
A

B

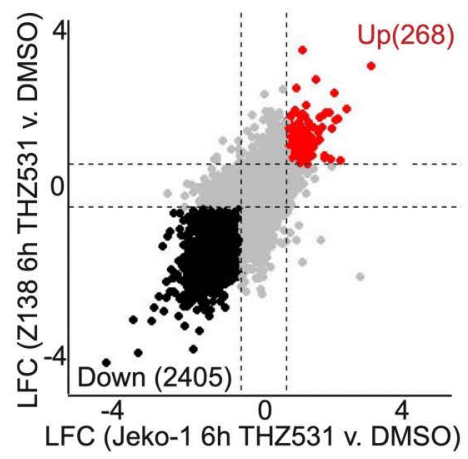

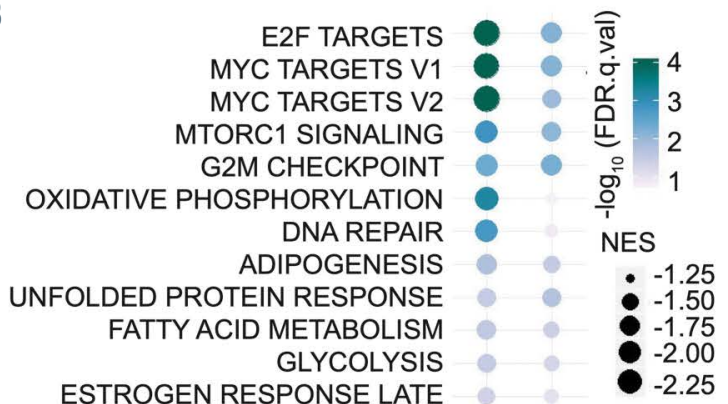

Jeko-1 Z138

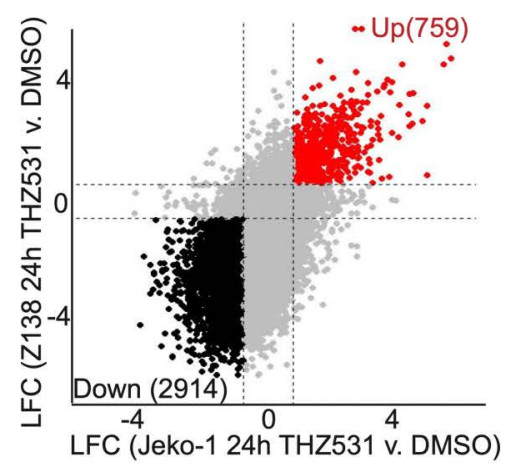

C

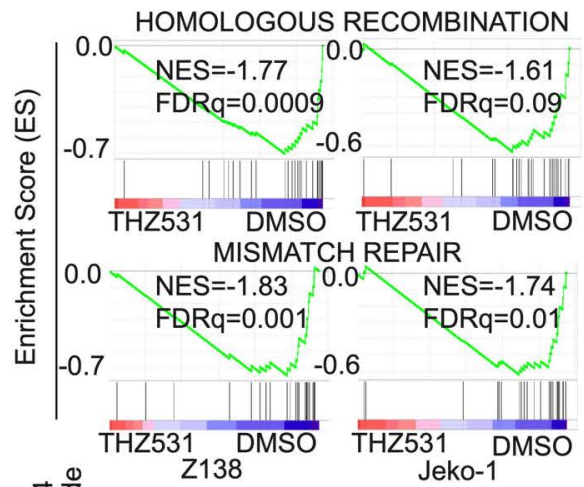

D
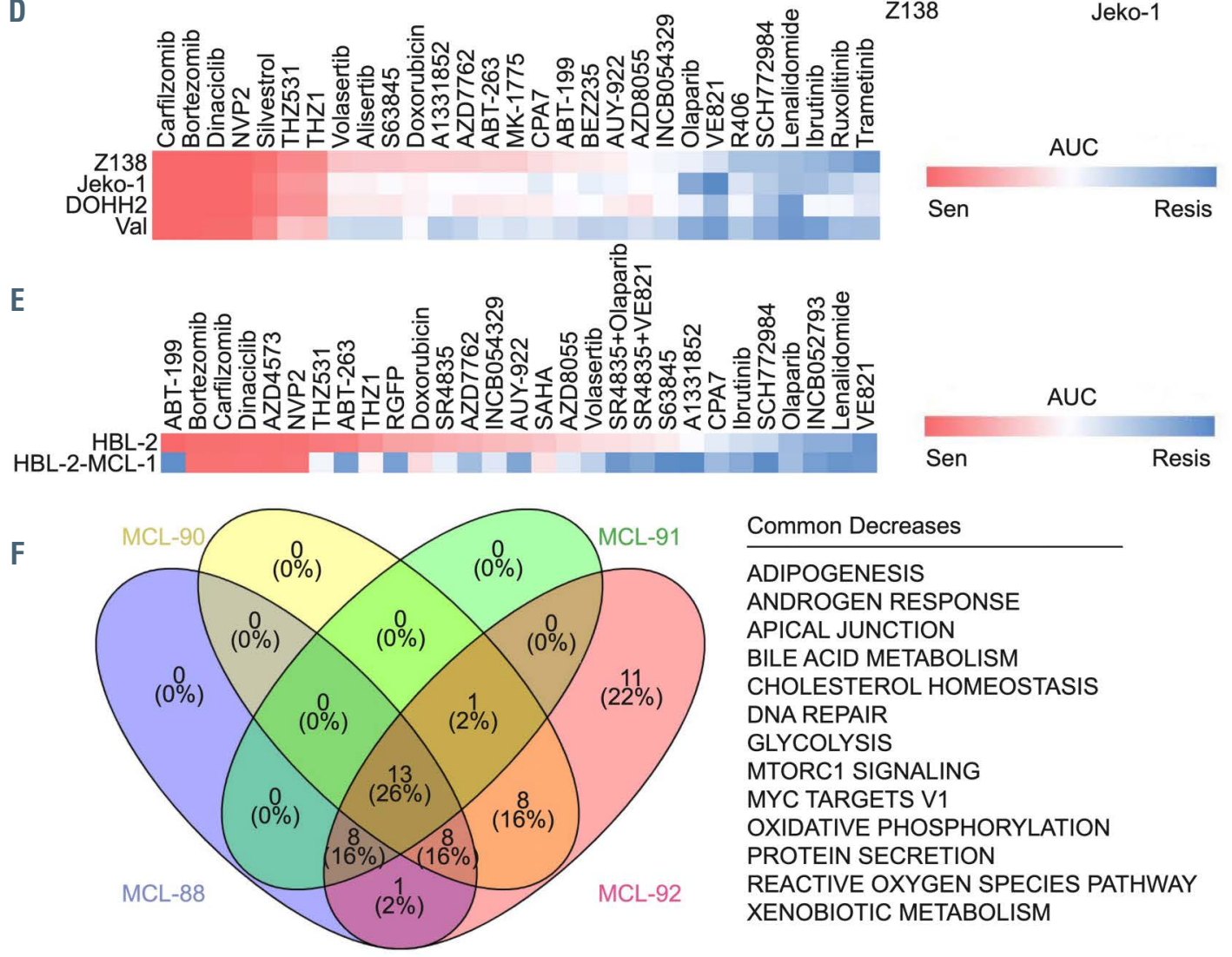

Common Decreases

ADIPOGENESIS

ANDROGEN RESPONSE

APICAL JUNCTION

BILE ACID METABOLISM

CHOLESTEROL HOMEOSTASIS

DNA REPAIR

GLYCOLYSIS

MTORC1 SIGNALING

MYC TARGETS V1

OXIDATIVE PHOSPHORYLATION

PROTEIN SECRETION

REACTIVE OXYGEN SPECIES PATHWAY

XENOBIOTIC METABOLISM

\footnotetext{
Figure 3. Transcriptomic analysis of THZ531-sensitive cells. (A) Transcriptome changes occurring in common in Z138 and Jeko-1 cells treated with THZ531 after 6 $\mathrm{h}$ (left) and $24 \mathrm{~h}$ (right). Red: upregulated genes, black: downregulated genes. LFC: $\log _{2}$ fold change cut-off of log 2 (1.5), $P$-value cutoff of 0.05 . Three biologically independent samples. (B) Dot plot of hallmark pathways that, from gene set enrichment analysis (GSEA), are negatively enriched in both Z138 and Jeko-1 cell lines treated with THZ531 after $24 \mathrm{~h}$. Larger dot sizes indicate a more negative enrichment score. Color scale represents significance. Genes are ranked according to their expression fold change after treatment. NES: normalized enrichment score; FDR: false discovery rate. (C) GSEA enrichment curves of KEGG pathways negatively enriched in both Z138 and Jeko-1 after THZ531 treatment. (D) Drug sensitivity shown as a heatmap of areas under the curves (AUC) calculated from dose-response curves obtained from cell viability assays performed in Z138, Jeko-1, DOHH2 and Val cell lines for each indicated drug administered for 72 h. (E ) Drug sensitivity shown as a heatmap of AUC calculated from dose-response curves obtained from image-based cell viability assays performed in HBL-2 cells with and without MCL1 overexpression for each indicated drug after $72 \mathrm{~h}$ of treatment. (F) Venn diagram of significantly negatively enriched GSEA hallmarks gene sets (NES <0, FDR $<0.25$ ) in mantle cell lymphoma primary patients' samples treated with $500 \mathrm{nM}$ THZ531 for $12 \mathrm{~h}$. Pathways negatively enriched in common in all four samples are listed. Data shown in (D and $\mathrm{E}$ ) are representative of at least three independent experiments.
} 
A

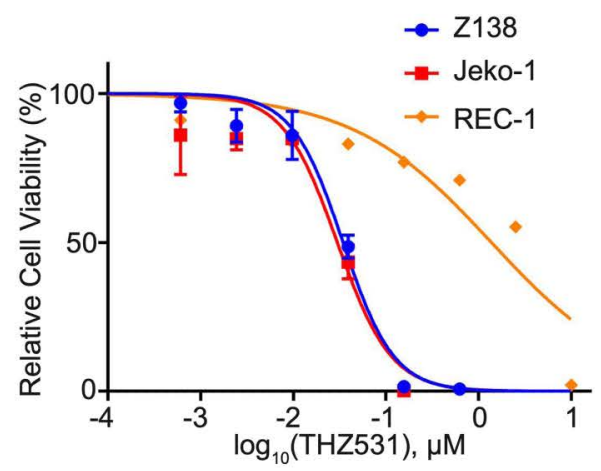

C

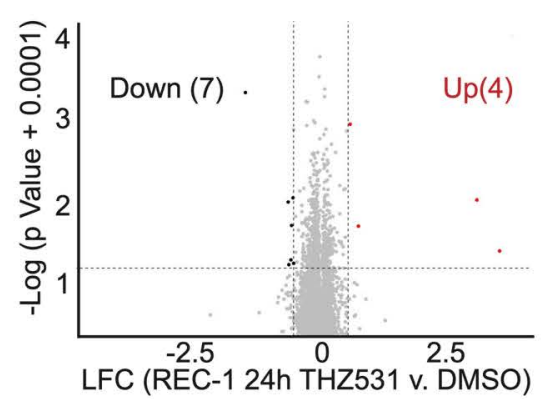

E

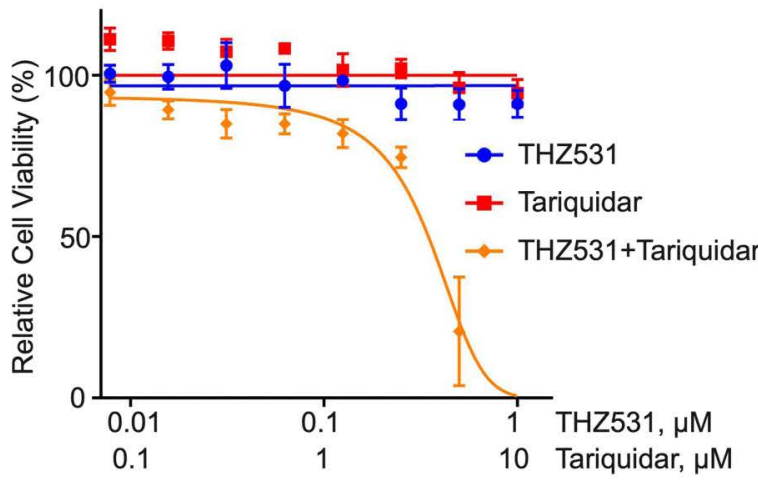

B

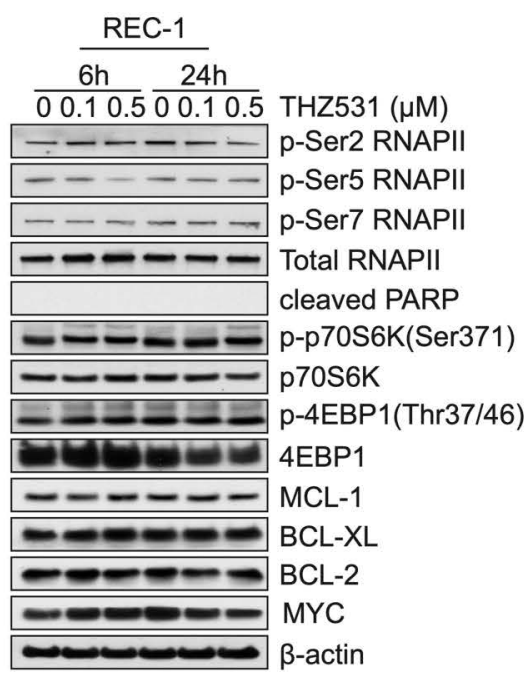

D

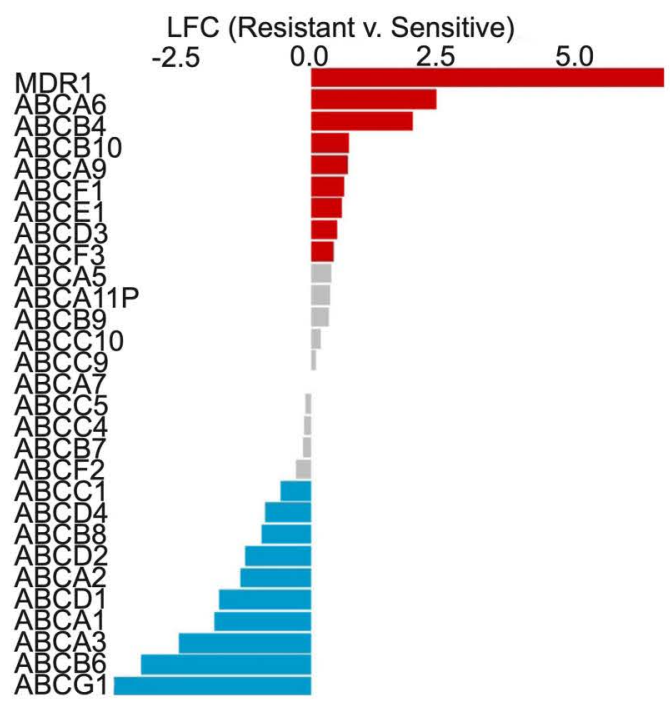

F
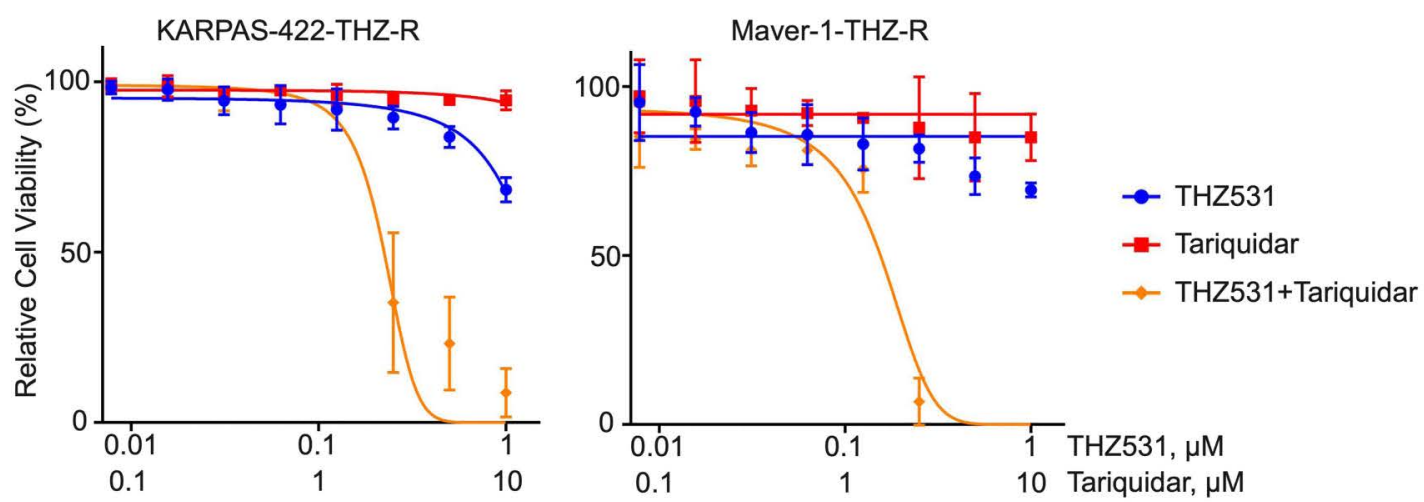

Figure 4. MDR1 upregulation drives resistance to THZ531 in mantle cell lymphoma and other aggressive B-cell lymphomas. (A) Dose-response curves of Z138, Jeko-1 and REC-1 cell lines after $72 \mathrm{~h}$ of treatment with THZ531. Data are shown as mean \pm standard deviation (SD) of three technical replicates for each cell line. (B) Western blot analysis of phosphorylation of RNAPII in Ser2, 5 and 7, RNAPII, cleaved PARP, phosphor-p70S6K, p70S6K, phosphor-4EBP1, 4EBP1, MCL-1, BCL$\mathrm{XL}, \mathrm{BCL}-2$ and MYC protein expression in REC-1 cells treated with the indicated doses of THZ531 at different time points. (C) Volcano plot showing no changes at the mRNA level after THZ531 treatment for $24 \mathrm{~h}$ relative to treatment with dimethylsulfoxide (DMSO) in REC-1 cells. Red: upregulated genes, black: downregulated genes. LFC: $\log _{2}$ fold change cutoff of $\log _{2}(1.5), P$-value cutoff of 0.05 . Three biologically independent samples. (D) Bar plot of differential expression of drug pump genes

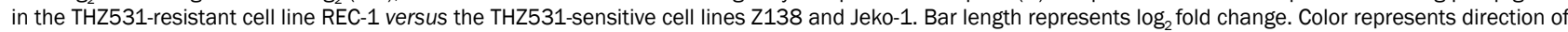
expressions change where red represents genes with $\log _{2}$ fold change greater than 1.5 and blue represents genes with log ${ }_{2}$ fold change less than 1.5 . (E) Doseresponse curves of REC-1 cells after $72 \mathrm{~h}$ of treatment with different doses of THZ531, tariquidar, or THZ531+tarquidar. Data are shown as mean \pm SD of three technical replicates for each cell line. (F) Dose-response curves of the KARPAS-422-THZ-R and Maver-1-THZ-R cell lines after $72 \mathrm{~h}$ of treatment with different doses of THZ531, tariquidar, or THZ531+tariquidar. Data are shown as mean \pm SD of three technical replicates for each cell line. Data shown in (A, E and F) are representative of at least three independent experiments. 
to these agents inevitably arises. Given that a recent study showed that $\mathrm{ABC}$ transporters mediate resistance to a THZ series of transcriptional CDK inhibitors, ${ }^{29,30}$ we next determined the role of MDR1 in de novo and acquired THZ531 resistance. Although sensitive cells had $\mathrm{IC}_{50}$ values in the nanomolar range, the de novo resistant line REC-1 had an IC50 of $1.63 \mu \mathrm{M}$ (Figure 4A). Using the same functional biochemical and pharmacogenomic approaches as those used for the THZ531-sensitive lines, we investigated the molecular pathways and mechanisms responsible for resistance
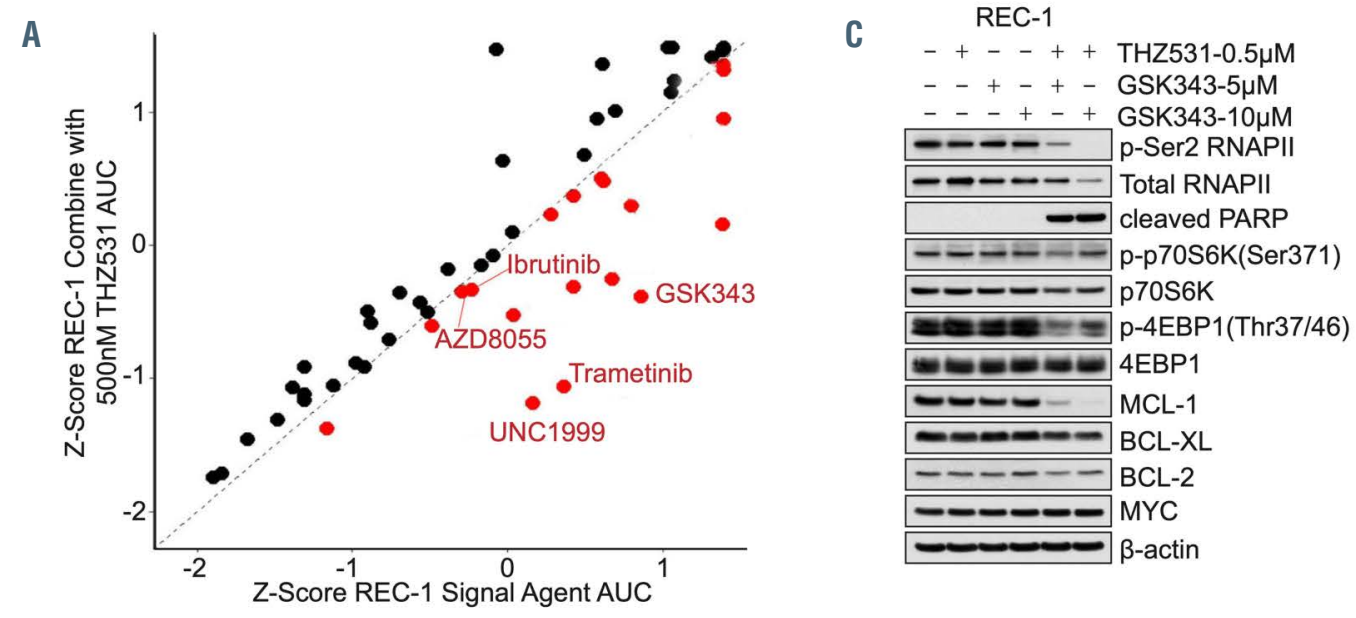

B
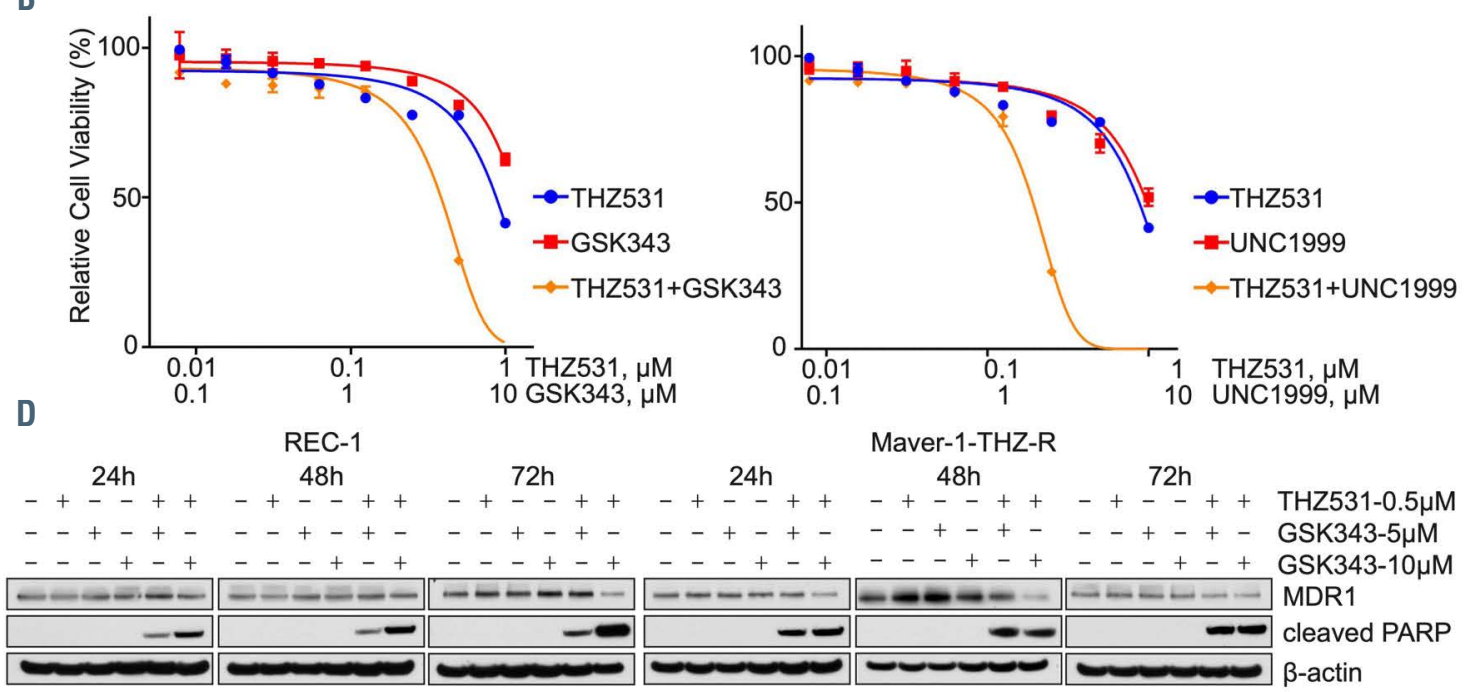

E

REC-1

KARPAS-422-THZ-R

Maver-1-THZ-R

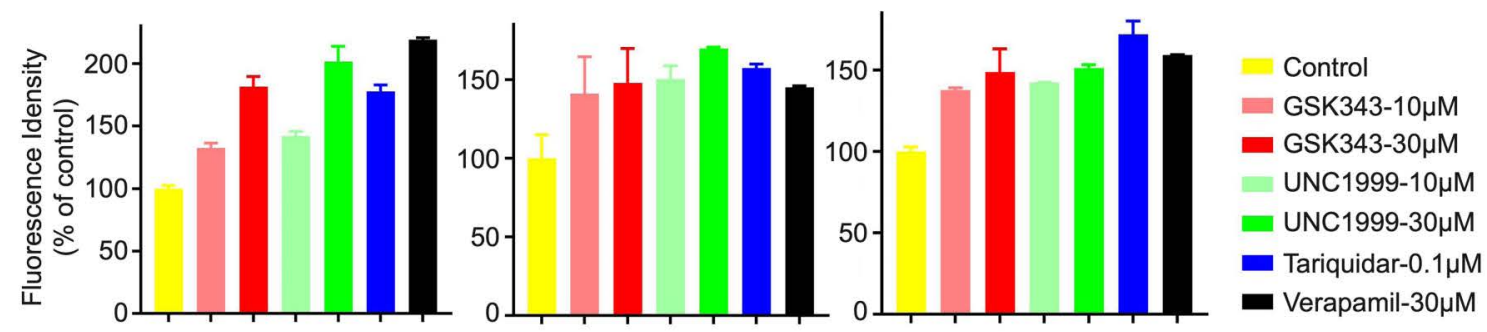

Figure 5. EZH2 inhibitors restored sensitivity to THZ531 in THZ531-resistant cells by competing with THZ531 for MDR1. (A). Z-Scores of normalized areas under the curve (AUC) of drug response curves from a high-throughput small-molecule drug screen performed in REC-1 cells for each indicated drug alone or combined with 500 nM THZ531. Selected compounds that had more potent effects upon combination with THZ531 are highlighted in red. (B) Left: dose-response curves of REC-1 cells after $72 \mathrm{~h}$ of treatment with different doses of THZ531, GSK343, or THZ531+GSK343. Right: dose-response curves of REC-1 cells after $72 \mathrm{~h}$ of treatment with different doses of THZ531, UNC1999, or THZ531+UNC1999. Data are shown as mean \pm standard deviation of three technical replicates for each cell line. (C) Western blot analysis of phosphorylation of RNAPII in Ser2, RNAPII, cleaved PARP, phosphor-p70S6K, p70S6K, phosphor-4EBP1, 4EBP1, MCL-1, BCL-XL, BCL-2 and MYC protein expression in REC-1 cells after $24 \mathrm{~h}$ of treatment with dimethylsulfoxide (DMSO) or indicated doses of THZ531 and/or GSK343. (D) Western blot analysis of MDR1 and cleaved PARP protein expression in REC-1 (left) and Maver-1-THZ-R (right) cell lines after treatment with DMSO or the indicated doses of THZ531 and/or GSK343 at different time points. (E) REC-1 (left), KARPAS-422-THZ-R (middle) and Maver-1-THZ-R (right) cells were treated with different drugs at the indicated doses for $12 \mathrm{~h}$. P-gp activity was determined using a fluorimetric MDR assay kit. The P-gp inhibitor verapamil ( $30 \mu \mathrm{M})$ served as a positive control. Results are expressed as mean \pm standard error of mean. Data shown in $(B)$ are representative of at least three independent experiments. 
A
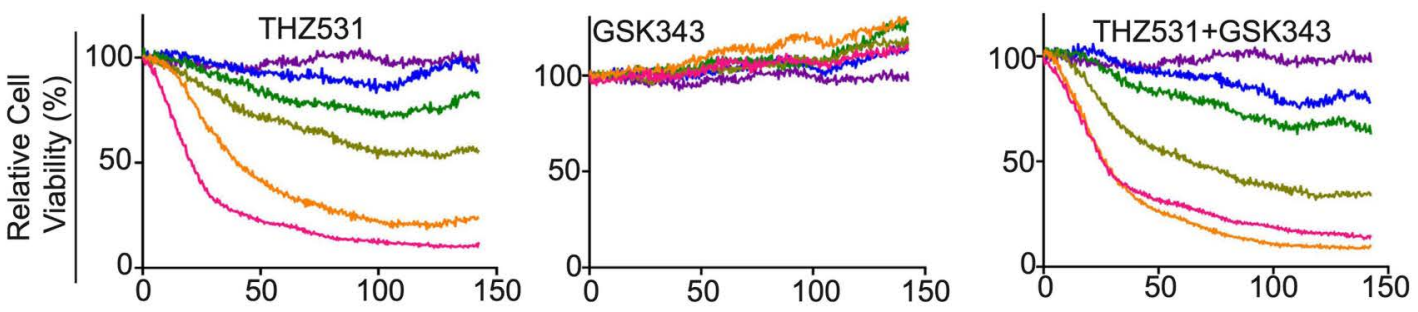

Time (h)

$$
\begin{array}{lllllll}
\text { THZ531: } & -3 \mu \mathrm{M} & -1 \mu \mathrm{M} & -0.33 \mu \mathrm{M} & -0.11 \mu \mathrm{M} & -0.04 \mu \mathrm{M} & - \text { DMSO } \\
\text { GSK343: } & -10 \mu \mathrm{M} & -3.33 \mu \mathrm{M} & -1.11 \mu \mathrm{M} & -0.37 \mu \mathrm{M} & -0.12 \mu \mathrm{M} & -\mathrm{DMSO}
\end{array}
$$

B

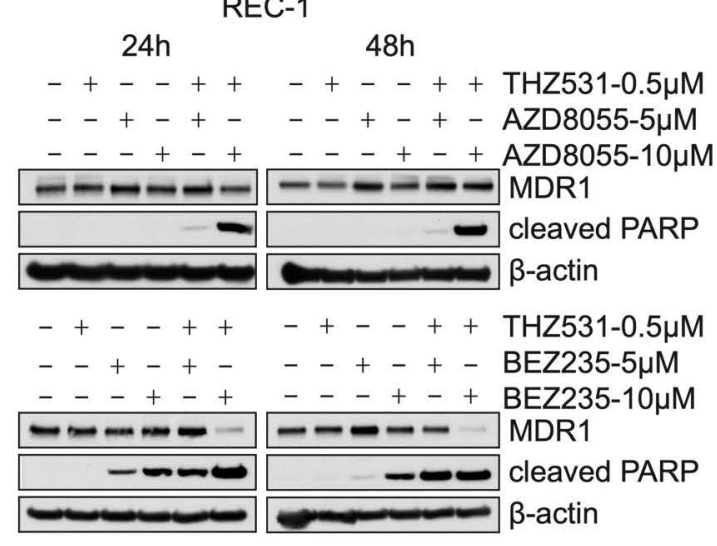

REC-1

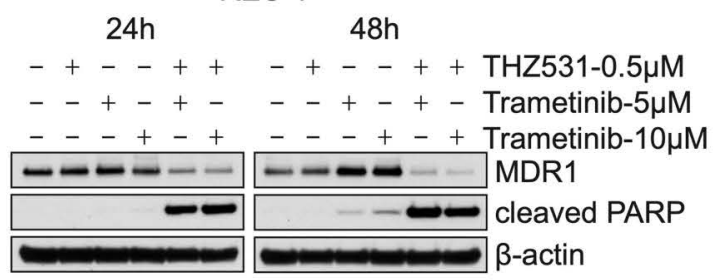

C

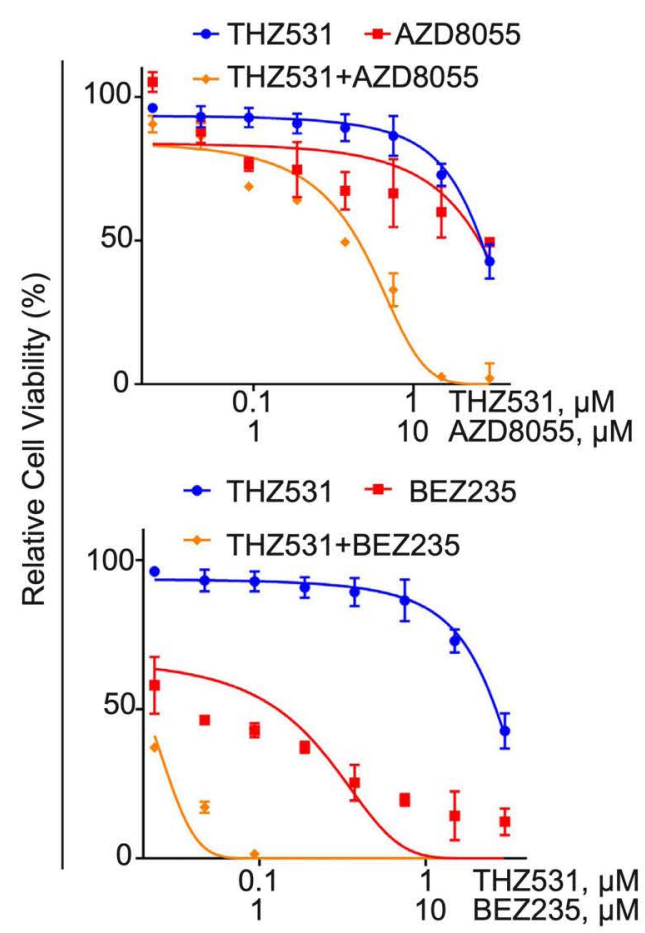

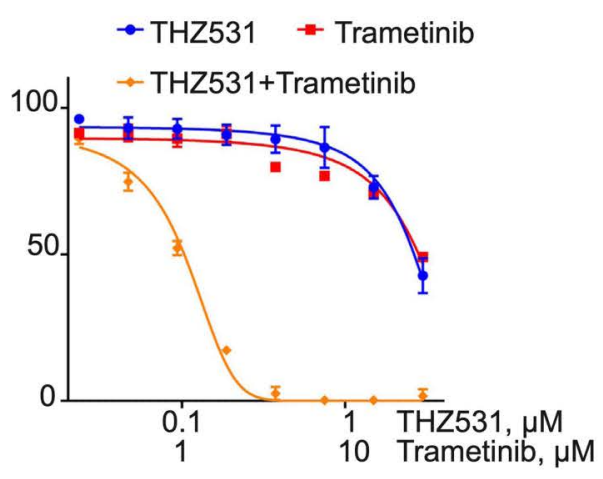

Figure 6. Activation of MEK-ERK and PI3K-AKT-mTOR pathways contributes to MDR1 upregulation in THZ531-resistant cells. (A) Image-based cell-viability assays of primary MCL samples, cells $\left(3 \times 10^{6}\right)$ cells were seeded in a 384-well plate with extracellular matrix and lymphoma stromal cells. THZ531 and GSK343 at five serial diluted concentrations were added to the medium, and the plate was continuously imaged every 30 min for $144 \mathrm{~h}$. All images were analyzed using a digital imaging analysis algorithm to detect cell viability based on membrane motion, and changes in viability were quantified by area under the curve (AUC). (B) Western blot of MDR1 and cleaved PARP protein expression in REC-1 cells after $24 \mathrm{~h}$ or $48 \mathrm{~h}$ of treatment with dimethylsulfoxide or indicated doses of THZ531 and/or AZD8055, BEZ235, or trametinib. (C) Dose-response curves of the REC-1 cells after treatment for $72 \mathrm{~h}$ with different doses of THZ531 and/or AZD8055, BEZ235 and trametinib. Data are shown as mean \pm standard deviation of three technical replicates for each cell line. Data shown in (C) are representative of at least three independent experiments. 
to THZ531. Western blotting revealed that, in contrast to THZ531-sensitive lines, the resistant REC-1 cells were refractory to THZ531 treatment and THZ531 failed to change the expression of MCL-1, MYC, and 4EBP1, PARP cleavage as well as all Ser2 phosphorylation of RNAPII (Figure 4B). Next, to investigate the cellular pathways responsible for THZ531 resistance, we analyzed the transcriptome changes after THZ531 treatment in REC-1. As illustrated in Figure 4C, in contrast to cells that were responsive to THZ531, THZ531 treatment for $24 \mathrm{~h}$ at a dose $(100 \mathrm{nM})$ that was highly potent in THZ531-sensitive lines did not induce gene expression changes in REC1. We then compared the differential gene expression between THZ531-sensitive cell lines (Z138, Jeko-1) and a THZ531-resistant one (REC-1). Among drug transport genes identified by RNA-sequencing, the level of MDR1 transcript was dramatically higher in REC-1 cells than in Z138 and Jeko-1 cells (Figure 4D). Moreover, quantitative reverse transcriptase polymerase chain reaction analysis revealed greater MDR1 abundance in REC-1 cells (Online Supplementary Figure S4A). To study the functional role of MDR1 in resistance to THZ531, we tested whether inhibition of MDR1 by its specific inhibitor (tariquidar) could overcome THZ531 resistance. As shown in Figure 4E, tariquidar overcame THZ531 resistance and synergized with THZ531 in the suppression of cell viability in REC1 cells. In addition, we developed two cell lines, KARPAS422-THZ-R and Maver-1-THZ-R (Online Supplementary Figure S4B), with resistance to THZ531: "acquired" by chronic exposure to escalating doses of THZ531 over 3 months. In line with cells with de novo drug resistance, the THZ531-resistant KARPAS-422 and Maver-1 cells exhibited higher MDR1 expression at both the mRNA and protein levels (Online Supplementary Figure S4C). Cell viability of both cell lines was dramatically reduced when exposed to the combination of THZ531 with tariquidar (Figure $4 \mathrm{~F})$. Together, these data support that MDR1 upregulation drives THZ531 resistance in MCL and other aggressive Bcell lymphomas.

\section{EZH2 inhibitors restored sensitivity to THZ531 in THZ531-resistant cells by competing with THZ531 for MDR1}

To define the molecular determinants that drive resistance to THZ531, reverse this resistance and enhance THZ531 activity in THZ531-resistant cell lines, we performed drug screen assays on REC-1 against a set of epigenetic modifiers and kinase inhibitors in the presence or absence of THZ531 as an "anchor" in our drug screen platform. Using AUC to quantify the effect and potency of each single and combination treatment, we identified the inhibitors that enhanced THZ531 activity and ranked the combination potency by the differential killing effect between drug combinations and single inhibitors. Inhibitors found to enhance the potency of THZ531 included MEK, BTK, mTOR and EZH2 inhibitors. Among them, the drug screen assay identified that inhibitors targeting EZH2 in combination with THZ531 had the greatest increased potency against REC-1 cells (Figure 5A, Online Supplementary Figure S5A). Indeed, cell viability assays revealed that the combination of EZH2 inhibitors (GSK343 or UNC1999) with THZ531 had synergistic effects against cell survival in REC-1 cells (Figure 5B). Mechanistically, western blots revealed that neither EZH2 inhibitor nor THZ531 alone had an effect on Ser2 phosphorylation of RNAPII, p-p70S6K, p-4EBP1, MCL-1 or PARP cleavage (Figure 5C, Online Supplementary Figure $S 5 B)$. However, the combination of either EZH2 inhibitor with THZ531 triggered dramatic decreases of Ser2 phosphorylation of RNAPII, p-p70S6K, p-4EBP1 and MCL-1 levels, and significantly increased PARP cleavage (Figure 5C, Online Supplementary Figure S5B). Consistently, synergistic effects of THZ531 combined with GSK343 or UNC1999 on cell viability were also observed in the THZ531-resistant cell lines KARPAS-422-THZ-R and Maver-1-THZ-R (Online Supplementary Figure S5C).

Next, to investigate the mechanisms by which EZH2 inhibition reinstated THZ531 sensitivity in resistant cells, we examined changes in MDR1 protein expression upon EZH2 inhibitor treatment in THZ531-resistant cells. As shown in Figure 5D, although treatments with any single agent had no effect on MDR1 protein expression, EZH2 inhibitor significantly sensitized REC-1 cells to THZ531 treatment, as measured by PARP cleavage. In contrast, EZH2 knockdown through shRNA showed that EZH2 downregulation had no effect on basal MDR1 expression and failed to reverse THZ531-induced downstream signaling via RNAPII-Ser2, MCL-1 and MYC in REC-1 cells (Online Supplementary Figure S5D). These data suggest that the potency enhancing effects of GSK343 and UNC1999 were not due to biological EZH2 regulation on either MDR1 or THZ531 signaling. Thus, to further reveal the underlying mechanism of MDR1 modulation by GSK343 and UNC1999, we performed P-gP-Glo assays, which served to elucidate whether GSK343 and UNC1999 are direct MDR1 inhibitors or substrates in REC-1, KARPAS422-THZ-R and Maver-1-THZ-R cells. GSK343 and UNC1999 were confirmed not to be direct inhibitors, but strong MDR1 substrates, comparable to verapamil (Figure $5 \mathrm{E})$. Thus, the data support that EZH2 inhibitors GSK343 and UNC1999 function by competitively binding to MDR1.

\section{Activation of MEK-ERK and PI3K-AKT-mTOR pathways contribute to MDR1 upregulation in THZ531-resistant cells}

We next systematically tested for synergistic combinations of THZ531 with EZH2 inhibitors in the sensitive as well as resistant cells to validate the combination as an effective strategy to overcome and block emergent resistance in primary samples. Importantly, in the innately THZ531-resistant primary samples, treatment with EZH2 inhibitors sensitized the cells to THZ531 (Figure 6A), strengthening the rationale to combine THZ531 and EZH2 inhibitors in the upfront setting.

Given that the drug screen on REC-1 cells showed inhibitors of MEK and AKT sensitized REC-1 to THZ531 treatment, we hypothesized that activation of the MEKERK and PI3-AKT-mTOR pathways in REC-1 regulated MDR1 expression and affected the efficacy of THZ531. As shown in Figure $6 \mathrm{~B}$, inhibition of MEK with trametinib and PI3K-AKT with either AZD8055 or BEZ235 induced a significant decrease of the abundance of MDR1 protein in REC-1 cells. Accordingly, combined treatment of THZ531 with trametinib, AZD8055 or BEZ235 all induced a synergistic effect on REC-1 cell viability, as measured by MTT assays (Figure 6C). Overall, these data imply that activation of MEK-ERK and PI3K-AKT-mTOR pathways contributes to MDR1 upregulation in THZ531resistant cells. 


\section{Discussion}

MCL is an incurable form of lymphoma and the median overall survival of patients with this malignancy is only 4 to 5 years. Its clinical course usually involves sequential relapses, with no response to current standard therapies. MYCassociated large B-cell lymphoma is one of the most aggressive lymphomas. Patients often experience relapsed or refractory disease after an initial response to first-line therapy and the majority of those with MYC-associated B-cell lymphomas succumb to their disease.

CDK12 is a transcriptional CDK that complexes with cyclin $\mathrm{K}$ to mediate gene transcription by phosphorylating RNAPII. CDK12 has been demonstrated to specifically upregulate the expression of genes involved in response to DNA damage, stress and proliferation, as well as mRNA processing and cell surviva ${ }^{17}$ by directly phosphorylating pre-mRNA processing factors, which induces premature cleavage and polyadenylation and a loss of expression of long genes that participate in the DDR. ${ }^{18,19}$ An increasing number of studies have highlighted CDK12 as a therapeutic target for cancer. Inhibition of transcriptional CDK could be an effective strategy to overcome resistance to targeted therapies, including erlotinib and crizotinib. Numerous other studies have identified specific genetic or cellular contexts that confer enhanced sensitivity to CDK12 inhibition, including MYC dependency. In this study, we aimed to explore CDK12 as a novel vulnerability for MCL and MYCassociated B-cell lymphomas. We further determined the role of CDK12-mediated transcription activation and associated pathways in cell survival and growth of MCL and MYC-associated B-cell lymphomas. Here, we report that the aggressive B-cell lymphomas are exquisitely sensitive to transcription-targeting drugs, particular to the covalent CDK12 inhibitor THZ531. By implementing pharmacogenomics and a cell-based drug screen, we found that THZ531 leads to inhibition of oncogenic transcriptional programs, especially the DDR pathway, MYC target genes and the mTOR-4EBP1-MCL-1 axis, contributing to lymphoma suppression ex vivo.

Importantly, we investigated molecular mechanisms involved in conferring resistance to THZ531 and examined whether combined inhibitors of CDK12 and EZH2 cooperatively reprogram transcription repression to overcome resistance to THZ531, and, ultimately, inhibit lymphoma growth and survival in aggressive B-cell malignancies. We demonstrate that MDR1 is overexpressed in cell lines with de novo and acquired resistance to THZ531, and that MDR1 overexpression results in THZ531 resistance via MDR1mediated export of THZ531. Our data implicate MDR1 as a therapeutic target to overcome THZ531 resistance. Additional testing with the chemically distinct CDK12 inhibitor SR-4835 revealed that MDR1 upregulation as an avenue of resistance in CDK12 inhibition is specific to THZ531 (data not shown). However, CDK12 inhibitors are known functional substrates for drug transport molecules, ${ }^{31}$ and accordingly, it has been reported that MDR1 upregulation contributes to resistance against other compounds with the THZ chemical backbone, including the CDK7 inhibitor THZ1. ${ }^{29}$ We then identified GSK343 and UNC1999, two EZH2 inhibitors, as potent drugs in preventing MDR1-mediated export of THZ531 in lymphoma cells. The combination of GSK343 or UNC1999 with THZ531 exhibited synergistic efficacy against MCL and aggressive B-cell lymphomas. We further showed that GSK343 and UNC1999 inhibited MDR1 efflux by competing with THZ531 for MDR1, consistent with previous reports suggesting that GSK343 and UNC1999 are potential MDR1 substrates. ${ }^{32}$ Thus, GSK343 and UNC1999 have a broad drug-sensitizing potential for lymphoma therapy. Furthermore, we also demonstrated that activation of MEK-ERK and PI3K-AKT-mTOR pathways contributes to MDR1 upregulation in THZ531-resistant cells. We identified that de novo and established acquired THZ531-resistant lymphoma cells are associated with over-activation of MEK-ERK and PI3K-AKT-mTOR pathways, contributing to upregulation of MDR1 protein. Intriguingly, EZH2 inhibitors reversed THZ531 resistance by competitive inhibition of MDR1 and, in combination with THZ531, synergistically inhibited MCL and MYC-associated lymphoma growth in vitro. Our study indicates that CDK12 inhibitors, both alone and together with EZH2 inhibitors, offer promise as a novel treatment strategy that can be an effective approach for MYC-dependent lymphomas and MCL. Furthermore, GSK343 and UNC1999 can also reverse drug resistance that has developed from MDR1 upregulation. Thus, GSK343 and UNC1999 can be used together with other MDR1-induced drugs such as doxorubicin and carfilzomib to overcome drug resistance for greater and longerlasting efficacy in patients with aggressive B-cell lymphomas.

\section{Disclosures}

No conflicts of interest to disclose.

\section{Contributions}

$J T$ and $X Z$ conceived and designed the study; JG, MYW, YR, TLi, JCY and TLw performed experiments, and collected and assembled the data; JG, MYW, XZ, RY and JT analyzed and interpreted the data; JT, JG and $M W$ wrote, reviewed and/or revised the manuscript; EMS, KHS, BDS, DRD and JT provided administrative, technical or material support.

\section{Funding}

This work was supported in part by grants from the National Cancer Institute CA241713, CA233601, and CA234519 (to JT), a grant from the Lymphoma Research Foundation (to JT) and by funds from Florida State Live Like Bella Pediatric Cancer Research Initiative to the H. Lee Moffitt Cancer Center \& Research Institute.

\section{References}

1. Nilsson JA, Cleveland JL. Myc pathways provoking cell suicide and cancer. Oncogene. 2003;22(56):9007-9021.

2. Dave SS, Fu K, Wright GW, et al. Molecular diagnosis of Burkitt's lymphoma. N Engl J Med. 2006;354(23):2431-2442.

3. Hartmann EM, Ott G, Rosenwald A.
Molecular biology and genetics of lymphomas. Hematol Oncol Clin North Am. 2008;22(5):807-823

4. Slack GW, Gascoyne RD. MYC and aggressive B-cell lymphomas. Adv Anat Pathol. 2011;8(3):219-228.

5. Ren Y, Bi C, Zhao X, et al. PLK1 stabilizes a MYC-dependent kinase network in aggressive B cell lymphomas. J Clin Invest.
2018;128(12):5517-5530

6. Hnisz D, Schuijers J, Lin CY, et al Convergence of developmental and oncogenic signaling pathways at transcriptional super-enhancers. Mol Cell. 2015;58(2):362 370.

7. Loven J, Hoke HA, Lin CY, et al. Selective inhibition of tumor oncogenes by disruption of super-enhancers. Cell. 2013;153(2):320 
334

8. Winter GE, Mayer A, Buckley DL, et al. BET bromodomain proteins function as master transcription elongation factors independent of CDK9 recruitment. Mol Cell. 2017; 67(1):5-18.

9. Ferguson FM, Gray NS. Kinase inhibitors: the road ahead. Nat Rev Drug Discov. 2018; 17(5):353-377.

10. Smith E, Lin C, Shilatifard A. The super elongation complex (SEC) and MLL in development and disease. Genes Dev. 2011; 25(7):661-672.

11. He N, Liu M, Hsu J, et al. HIV-1 Tat and host AFF4 recruit two transcription elongation factors into a bifunctional complex for coordinated activation of HIV-1 transcription. Mol Cell. 2010; 38(3):428-438.

12. Chipumuro E, Marco E, Christensen CL, et al. CDK7 inhibition suppresses superenhancer-linked oncogenic transcription in MYCN-driven cancer. Cell. 2014; 159(5): 1126-1139.

13. Christensen CL, Kwiatkowski N, Abraham $\mathrm{BJ}$, et al. Targeting transcriptional addictions in small cell lung cancer with a covalent CDK7 inhibitor. Cancer Cell. 2014; 26(6):909-922.

14. Wang Y, Zhang T, Kwiatkowski N, et al. CDK7-dependent transcriptional addiction in triple-negative breast cancer. Cell. 2015; 163(1):174-186

15. Pelish HE, Liau BB, Nitulescu II, et al. Mediator kinase inhibition further activates super-enhancer-associated genes in AML. Nature. 2015;526(7572):273-276.

16. Zhao X, Ren Y, Lawlor M, et al. BCL2 amplicon loss and transcriptional remodeling drives ABT-199 resistance in B cell lymphoma models. Cancer Cell. 2019;35(5):752 766.

17. Lui GYL, Grandori C, Kemp CJ. CDK12: an emerging therapeutic target for cancer. J Clin Pathol. 2018;71(11):957-962.

18. Dubbury SJ, Boutz PL, Sharp PA. CDK12 regulates DNA repair genes by suppressing intronic polyadenylation. Nature. 2018; 564(7734):141-145.

19. Krajewska M, Dries R, Grassetti AV, et al CDK12 loss in cancer cells affects DNA damage response genes through premature cleavage and polyadenylation. Nat Commun. 2019;10(1):1757.

20. Iniguez $A B$, Stolte $B$, Wang EJ, et al. EWS/FLI confers tumor cell synthetic lethality to CDK12 inhibition in Ewing sarcoma. Cancer Cell. 2018;33(2):202-216.

21. Johnson SF, Cruz C, Greifenberg AK, et al CDK12 inhibition reverses de novo and acquired PARP inhibitor resistance in BRCA wild-type and mutated models of triple-negative breast cancer. Cell Rep. 2016; 17(9):2367-2381.

22. Jares P, Colomer D, Campo E. Molecular pathogenesis of mantle cell lymphoma. J Clin Invest. 2012;122(10):3416-3423.

23. Campaner S, Amati B. Two sides of the Myc-induced DNA damage response: from tumor suppression to tumor maintenance. Cell Div. 2012;7(1):6.

24. Rohban S, Campaner S. Myc induced replicative stress response: how to cope with it and exploit it. Biochim Biophys Acta. 2015;1849(5):517-524
25. Silva A, Jacobson T, Meads M, et al. An organotypic high throughput system for characterization of drug sensitivity of primary multiple myeloma cells. J Vis Exp. 2015;(101):e53070.

26. Silva A, Silva MC, Sudalagunta P, et al. An ex vivo platform for the prediction of clinical response in multiple myeloma. Cancer Res. 2017;77(12):3336-3351.

27. Zhao X, Lwin T, Silva A, et al. Unification of de novo and acquired ibrutinib resistance in mantle cell lymphoma. Nat Commun. 2017; 8:14920.

28. Wang J, Ye Q, She OB. New insights into 4EBP1-regulated translation in cancer progression and metastasis. Cancer Cell Microenviron. 2014;1(5):e331.

29. Gao Y, Zhang T, Terai H, et al. Overcoming resistance to the THZ series of covalent transcriptional CDK inhibitors. Cell Chem Biol. 2018;25(2):135-142.

30. Olson CM, Liang Y, Leggett $A$, et al. Development of a selective CDK7 covalent inhibitor reveals predominant cell-cycle phenotype. Cell Chem Biol. 2019:26(6):792-803

31. Cihalova D, Staud F, Ceckova M. Interactions of cyclin-dependent kinase inhibitors AT-7519, flavopiridol and SNS032 with $A B C B 1, A B C G 2$ and $A B C C 1$ transporters and their potential to overcome multidrug resistance in vitro. Cancer Chemother Pharmacol. 2015;76(1):105116.

32.Zhang P, de Gooijer MC, Buil LC, et al $A B C B 1$ and $A B C G 2$ restrict the brain penetration of a panel of novel EZH2-Inhibitors. Int J Cancer. 2015;137(8):2007-2018. 